\title{
The relation among critical ratios, critical bands, and intensity difference limens in the parakeet (Melopsittacus undulatus)
}

\author{
ROBERT J. DOOLING and MARGARET H. SEARCY \\ Rockefeller University, Field Research Center, Millbrook, New York 12545
}

\begin{abstract}
Intensity difference limens obtained for the parakeet using amplitude modulated pure tones indicate a Weber fraction $(\Delta \mathrm{I} / \mathrm{I})$ of about .23 to .43 over a range of five test frequencies. These values are lower than previous measures of intensity resolving power in this species and are in perfect agreement with previously published critical ratio and critical band data for the parakeet.
\end{abstract}

Bilger (1976) has recently suggested a resolution to the problem of "directly" measured critical bandwidths being 2.5 times wider than "indirectly" measured critical bandwidths as inferred from broadband masking data. Fundamental to the resolution of this issue is the notion that masking data and intensity discrimination data can often be treated interchangeably (Bos \& deBoer, 1966; Green, 1960; McGill \& Goldberg, 1968; Miller, 1947). Critical ratio and critical band data are in agreement if one accepts that the task involved in a critical ratio experiment is, as Bilger states, "to discriminate an intensive difference between a critical bandwidth of noise alone and a critical bandwidth of noise plus signal" (1976, p. 194). Thus, the somewhat arbitrary assumption that at masked threshold the signal power and the noise power in the critical bandwidth are equal is faulty. Since this line of reasoning accounts for the discrepancy between critical ratio and critical band data in the human, it would seem of interest to examine the relation among critical bands, critical ratios, and intensity difference limens in other species. As far as we know, all three of these measures are available for only three species: cat, mouse, and parakeet.

The problem of relating critical bandwidths with inferred bandwidths from critical ratio experiments, then, is that bandwidths estimated from broadband masking data are too narrow. In fact, they are too narrow by a factor equal to the Weber fraction for intensity. Otherwise stated, critical bandwidth = critical ratio $\times 1$ /Weber fraction. Data available for the cat at $1,000 \mathrm{~Hz}$ indicate a directly measured critical bandwidth of $345 \mathrm{~Hz}$ (Pickles, 1975), an inferred bandwidth of $144.5 \mathrm{~Hz}$ (Watson, 1963), and a Weber fraction for intensity $(\Delta \mathrm{I} / \mathrm{I})$ of .521 (Rabb \& Ades, 1946). The observed Weber fraction for intensity obtained from the difference limen experiment is slightly larger than one would expect from the critical ratio and critical band

This research supported by Public Health Service Grant MH 31165. data for the cat at $1,000 \mathrm{~Hz}$. Similarly, Ehret $(1975 \mathrm{~b}$, 1976) has shown for the mouse that bandwidths estimated from critical ratio experiments are about the same size as directly measured critical bands. This relation would lead one to expect a Weber fraction for intensity of about 1.0 for the mouse. Instead, intensity difference limens for the mouse show a Weber fraction of 2.0 (Ehret, 1975a). In the parakeet, also, the relation between directly measured critical bandwidths (Saunders, Denny, \& Bock, 1978) and inferred bandwidths from broadband masking experiments (Dooling \& Saunders, 1975a) would lead to an expected Weber fraction for intensity of about .40 , in contrast to the obtained Weber fraction of about 1.0 (Dooling \& Saunders, 1975b).

Figure 1 shows the relation between previously measured critical bands and critical ratios. The critical ratio

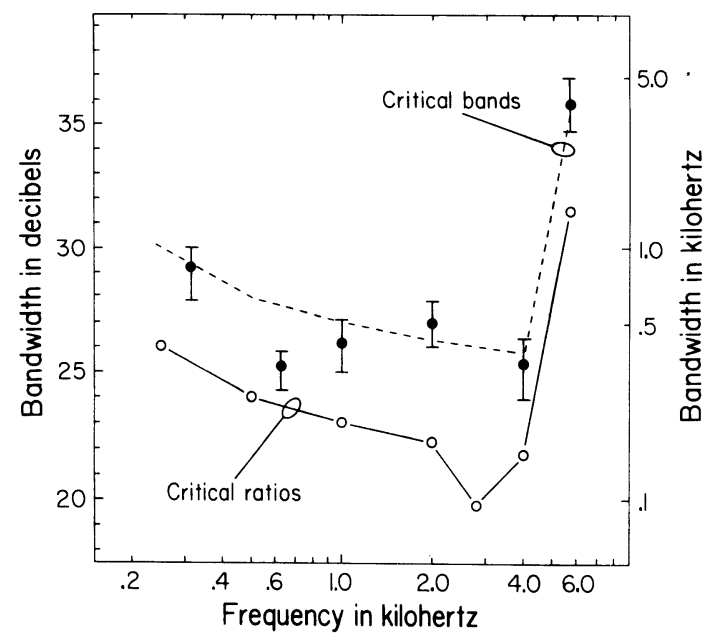

Figure 1. Comparison of critical ratio and critical band functions for the parakeet. Critical ratio data are replotted from Dooling and Saunders (1975a) and solid symbols are critical band data replotted from Saunders, Denny, and Bock (1978). Dotted line is the critical ratio function displaced upward by a factor of 2.5 (or $4 \mathrm{~dB}$ ). 
data are from Dooling and Saunders (1975a); the critical band data (solid points) are replotted from Saunders et al. (1978). The dashed line represents a displacement of the critical ratio curve upward by a factor of 2.5 (or about $4.0 \mathrm{~dB}$ ). This relation would lead one to expect a Weber fraction for intensity equal to: $W F=$ $\mathrm{CR}(\mathrm{Hz}) / \mathrm{CB}(\mathrm{Hz})$.

The magnitude and direction of the discrepancies between these three measures in each species suggests the possibility that differences in procedures for collecting masking data and intensity difference limen data are responsible. Specifically, it might be argued that intensity difference limens obtained by amplitude modulating a continuous signal would not only result in smaller difference limens but would also more closely approximate a simultaneous masking task than would intensity difference limens obtained using pulsed sinusoids in a repeating background paradigm. The present experiment measures intensity difference limens in the parakeet at five test frequencies using amplitudemodulated pure tones.

\section{METHOD}

The output of an oscillator was split and one part was passed through a programmable attenuator. The second part was added in phase to the output from this attenuator, permitting extremely accurate control of increments in intensity. The birds were tested in the presence of a continuous pure tone at a sensation level of $55 \mathrm{~dB}$ (Dooling \& Saunders, 1975a). A trial consisted of intensity increments in this signal having durations of $500 \mathrm{msec}$ and rise-fall times of $50 \mathrm{msec}$. Five such increments were presented at the rate of $1 \mathrm{~Hz}$.

The birds were trained and tested in a tubular restraining device described previously (Dooling, 1973). Failure to respond to the five intensity increments resulted in a buzzer and shock being paired with the stimulus until a correct response occurred. This instrumental avoidance procedure was used with the method of limits to measure intensity difference limens in two parakeets. The procedure was employed exactly as described before in measuring frequency difference limens (Dooling \& Saunders, 1975a), intensity difference limens (Dooling \& Saunders, 1975b), and duration difference limens (Dooling \& Haskell, 1978) in the parakeet. In all, intensity difference limens were obtained at the test frequencies of .5, 1.0, 2.0, 4.0 , and $5.7 \mathrm{kHz}$. Both directly measured critical bandwidths and inferred bandwidths from critical ratio measurements are available at these test frequencies for the parakeet.

\section{RESULTS}

Twenty final threshold determinations ( 10 from each bird) were available for each test frequency. The reciprocal of the Weber fraction for intensity was computed for each of the 20 threshold measures and multiplied by the critical ratio (in hertz) to obtain the critical bandwidth. These critical bandwidths are shown by the solid data points in Figure 2. As before, the critical ratio data are from Dooling and Saunders (1975a) and the critical band function is merely the critical ratio function displaced upward by a factor of 2.5 (or $4 \mathrm{~dB}$ ). As can be seen, the fit between the hypothetical critical band function and

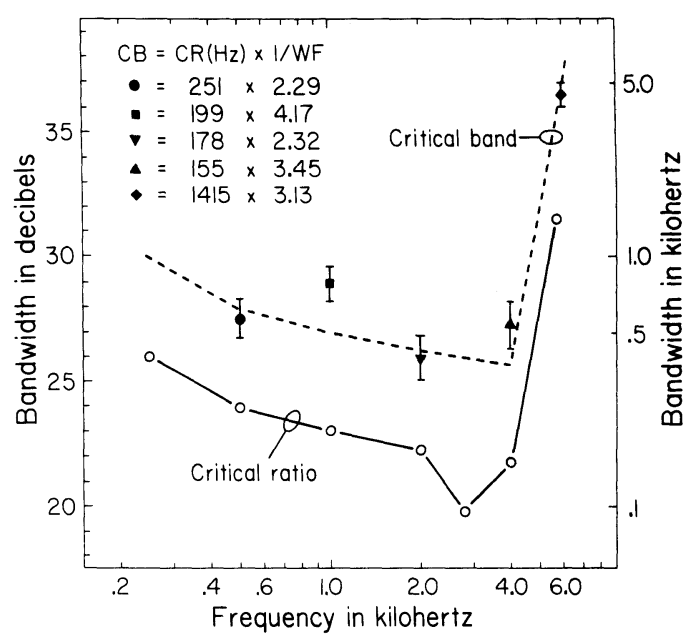

Figure 2. Comparison of critical ratio and critical band data of previous figure with the Weber fraction for intensity at five frequencies. Solid symbols are the critical ratio in hertz multiplied by the reciprocal of the Weber fraction for intensity at each frequency.

the critical bandwidths calculated from the product of the critical ratio and the reciprocal of the Weber fraction for intensity is quite good.

\section{DISCUSSION}

Both the critical ratio and the critical band functions for the parakeet show a pattern of masking as a function of frequency that is dramatically different from that observed in other species (including man). Historically, it has been argued that the critical band represents the mechanical frequency resolving power of the cochlea (Fletcher, 1940). It is generally accepted that the place of maximum displacement is organized logarithmically along the basilar membrane (Békésy, 1960) and, as a consequence, critical bands are viewed as representing constant distances along the basilar membrane for any one species (Ehret, 1975b; Greenwood, 1961, 1962; Watson, 1963).

While it is well established that at least in the chicken (and most probably in other birds) frequency is also organized logarithmically along the basilar papilla (Békésy, 1960), the peculiar shape of the critical ratio and critical band functions preclude an extension of the idea that critical bands represent equal distances along the parakeet basilar papilla (Dooling \& Saunders, 1975a). It is interesting in this regard that in spite of the dissociation between frequency mapping on the basilar papilla and critical band mapping on the basilar papilla, the relation between critical ratios, critical bands, and intensity difference limens shows the same pattern as that observed for man (Bilger, 1976). Several possibilities suggest themselves. First, in the frequency region of $2.8-4.0 \mathrm{kHz}$, the parakeet critical ratio function does increase at the rate of about $3 \mathrm{~dB} /$ octave, a pattern characteristic of the critical ratio functions of most other vertebrates. Perhaps frequency position and critical bandwidth have the expected relation over this very narrow range of frequencies but not for higher or lower frequencies. Second, it may be that the mechanism that accounts for critical band effects in the parakeet is simply not as peripheral (i.e., mechanical) as its counterpart in other vertebrates.

Regardless of the final resolution, the present data are clearly in support of the notion that in a masking situation involving broadband noise and a narrow signal, the task is one of detecting the difference in intensity between a critical band of noise alone and a critical band of noise plus signal. 


\section{REFERENCES}

BÉKÉsY, G. V. Experiments in hearing. New York: McGraw-Hill, 1960.

BILGER, R. C. A revised critical band hypothesis. In S. K. Hirsh, I. J. Eldredge, I. J. Hirsh, \& S. R. Silverman (Eds.), Hearing and Davis: Essays honoring Hollowell Davis. Saint Louis: Washington University Press, 1976.

Bos, C. E., \& DEBOER, E. Masking and discrimination. Journal of the Acoustical Society of America, 1966, 39, 708-715.

Dooling, R. J. Behavioral audiometry with the parakeet (Melopsittacus undulatus). Journal of the Acoustical Society of America, 1973, 53, 1757-1758.

Dooling, R. J., \& HASkell, R. J. Auditory duration discrimination in the parakeet (Melopsittacus undulatus). Journal of the Acoustical Society of America, 1978, 63, 1640-1642.

Dooling, R. J., \& SAUnders, J. C. Hearing in the parakeet (Melopsittacus undulatus): Absolute thresholds, critical ratios, frequency difference limens, and vocalizations. Journal of Comparative and Physiological Psychology, 1975, 88, 1-20. (a)

Dooling, R. J., \& SAUNDERs, J. C. Auditory intensity discrimination in the parakeet (Melopsittacus undulatus). Journal of the Acoustical Society of America, 1975, 58, 1308-1310. (b)

EHRET, G. Frequency and intensity difference limens and nonlinearities in the ear of the house mouse (Mus musculus). Journal of Comparative Physiology, 1975, 102, 321-336. (a)

EHRET, G. Masked auditory thresholds, critical ratios, and scales of the basilar membrane of the house mouse (Mus musculus). Journal of Comparative Physiology, 1975, 103, 329-341. (b)

EHRET, G. Critical bands and filter characteristics in the ear of the house mouse (Mus musculus). Biological Cybernetics, 1976, 24, 35-42.
Fletcher, H. Auditory patterns. Review of Modern Physics, 1940, 12, 47-65.

GreEn, D. M. Auditory detection of a noise signal. Journal of the Acoustical Society of America, 1960, 32, 121-131.

Greenwood, D. D. Critical bandwidth and the frequency coordinates of the basilar membrane. Journal of the Acoustical Society of America, 1961, 33, 1344-1356.

GREENwOOD, D. D. Approximate calculation of the dimensions of travelling wave envelopes in four species. Journal of the Acoustical Society of America, 1962, 34, 1364-1369.

McGill, W. J., \& Goldberg, J. P. A study of the near miss involving Weber's law and pure-tone intensity discrimination. Perception and Psychophysics, 1968, 4, 105-109.

Miller, G. A. Sensitivity to changes in the intensity of white noise and its relation to masking and loudness. Journal of the Acoustical Society of America, 1947, 19, 609-619.

Pickles, J. O. Normal critical bands in the cat. Acta Otolaryngology, 1975, 80, 245-254.

RAAB, D., \& ADES, H. Cortical and midbrain mediation of a conditioned discrimination of acoustic intensities. American Journal of Psychology, 1946, 59, 59-83.

Saunders, J. C., Denny, R. M., \& Bock, G. R. Critical bands in the parakeet (Melopsittacus undulatus). Journal of Comparative Physiology, 1978, 125, 359-365.

SChARF, B. Critical bands. In J. V. Tobias (Ed.), Foundations of modern auditory theory. New York: Academic Press, 1970.

Watson, C. S. Masking of tones by noise for the cat. Journal of the Acoustical Society of America, 1963, 35, 989-997.

(Received for publication January 31, 1979.) 